\title{
Effect of Music Training on Auditory Brainstem and Middle Latency Responses
}

\author{
Chul-Hee Choi ${ }^{1,2,3}$, Hea-Sung Cho ${ }^{1}$ \\ 'Department of Audiology and Speech-Language Pathology, The Graduate School of Health and Medical Industry, \\ Daegu Catholic University, Gyeongsan, Korea \\ ${ }^{2}$ Research Institute of Biomimetic Sensory Control, Daegu Catholic University, Gyeongsan, Korea \\ ${ }^{3}$ Catholic Hearing Voice Speech Center, Daegu Catholic University, Gyeongsan, Korea
}

\author{
Received: October 5, 2019 \\ Revised: October 11, 2019 \\ Accepted: October 13, 2019 \\ Correspondence: \\ Chul-Hee Choi, PhD \\ Department of Audiology and \\ Speech-Language Pathology, \\ Research Institute \\ of Biomimetic Sensory Control, \\ Catholic Hearing Voice Speech Center \\ Daegu Catholic University, \\ 13-13 Hayang-ro, Hayang-eup, \\ Gyeongsan 38430, Korea \\ Tel: +82-53-850-2541 \\ Fax: +82-53-850-3383 \\ E-mail: cchoi@cu.ac.kr
}

\begin{abstract}
Purpose: The auditory system has potentials to reorganize its structure and function in response to environmental changes such as training, experience, learning, injury, and disease. This is called neuroplasticity. A typical example of neuroplasticity is the music training, which demands cognitive and neural challenges resulting in enhanced auditory perception. This study investigated the effect of music training on auditory evoked responses, particularly auditory brainstem and middle latency responses. Methods: Forty college students consisting of twenty students with music training (musicians) and twenty students without music training (non-musicians) participated in the study. All participants have normal ranges in terms of pure tone audiometry, speech audiometry, and tympanometry. Auditory brainstem response (ABR) and auditory middle latency response (AMLR) from both ears were tested. Absolute latencies and amplitudes of waves in ABR and AMLR were obtained and analyzed. Results: The absolute latencies of wave I and $V$ significantly differed between musicians and non-musicians. They were shorter for musicians than for non-musicians. Significant differences were found in the interpeak latencies of wave III-V and I-V between musicians and non-musicians. They were also shorter in musicians than for non-musicians. In addition, there were only significant differences in the latency of $\mathrm{Na}$ in AMLR between musicians and non-musicians. It was shorter for a musician than for non-musician. Conclusion: ABR was more sensitive to the efficacy of the music training.
\end{abstract}

Key Words: Music training, Auditory brainstem response, Auditory middle latency response, Auditory evoked responses, Neuroplasticity.

\section{INTRODUCTION}

Musical performance is a highly complicated task which requires precise regulation and organization of the sensorimotor system under high order cognitive functions of the brain (Miyamae, 2018). Therefore, being a professional musician requires a lot of training and practice from childhood. It has been reported that it requires more than 10,000 hours by the age of 21 (Ericsson et al., 1993). Professionals who have been trained in music for a long time may be different in the structure and activity of the brain than those who do not. For example, professional violin players who require fine finger exercise have structurally a larger motor area of cerebrum than those with no

(c) This is an Open Access article distributed under the terms of the Creative Commons Attribution Non-Commercial License (https://creativecommons.org/licenses/by-nc/4.0) which permits unrestricted non-commercial use, distribution, and reproduction in any medium, provided the original work is properly cited. training (Gaser \& Schlaug, 2003). This indicates that long-term exercises and practices in specific fields change both the structure and function of the cerebrum. This is called neuroplasticity. Neuroplasticity denotes a variety of changes in the structure and function of the brain or the central nervous system in response to many environmental changes such as training, learning, experience, stimulus, injury, disease, and treatment. Music training requires complex multimodal skills such as auditory, visual, somatosensory as well as the motor system and relates to many brain functions like perception, action, cognition, emotion, learning, and memory (Pantev \& Herholz, 2011; Pantev et al., 2009). Therefore, music has been used as an ideal tool to investigate how the brain is working and how different brain functions interact.

Music relates primarily the auditory system which has potentials to reorganize its structure and function in response to mu- 
sic or music training. Structural and functional changes occur from the brainstem to primary and surrounding auditory cortices to areas involved in higher-order auditory cognition (Herholz \& Zatorre, 2012). The effect of music training on the brain structure and function was investigated by comparing brainstem and cortical auditory evoked potentials between musicians and non-musicians. Musicians showed more enhanced and better spectral analysis of linguistic pitch, higher similarities between brainstem responses of speech in quiet and noisy conditions, enhanced neural synchronization and brainstem encoding for defining characteristics of musical sequences, increased brainstem discrimination of closely related speech sounds, and unsusceptible age-related regression in neural timing compared to those in non-musicians (Bidelman \& Krishnan, 2010; Parbery-Clark et al., 2009; Parbery-Clark et al., 2011; Sanju \& Kumar, 2016; Strait et al., 2014; Wong et al., 2007). In addition, musicians showed shorter latency and greater amplitude of cortical auditory evoked potentials, more robust P2 and N1c auditory evoked potential, and positive effects of musical training and experience on the central auditory nerve system, compared to non-musicians (Sanju \& Kumar, 2016).

Music training is a major topic to assess neuroplasticity in brainstem and cortical areas with auditory evoked responses (AERs) as an objective measure of the auditory system. AERs are one of electrophysiological measures recording a series of electrical responses generated in the peripheral and central nervous systems in response to acoustical sounds (Anderson \& Jenkins, 2015). Therefore, this study investigated whether there are significant differences in auditory brainstem response (ABR) and auditory middle latency response (AMLR) between college students with and without music training.

\section{MATERIALS AND METHODS}

\section{Subjects}

Forty college students consisting of twenty students (musicians) with music training and twenty students without music

Table 1. Information of participants with music training $(n=20)$

\begin{tabular}{clc}
\hline Number of subject & Musical instruments & Music experience (yrs) \\
\hline 10 & Violin & $13.2 \pm 3$ \\
3 & Piano & $13.3 \pm 5.5$ \\
3 & Clarinet & $11.7 \pm 1.5$ \\
1 & Horn & 11 \\
1 & Percussion & 9 \\
1 & Viola & 14 \\
1 & Cello & 13 \\
\hline
\end{tabular}

training (non-musicians) participated in the study. Whether a subject is a musician or not was determined by the period of the musical training. The mean training years for the musician groups were $12.65 \pm 3$ (yrs) whereas the non-musician groups have no musical training. The participants' information of the musical instruments, the number of subjects, and the training years was listed in Table 1.

Testing by an otoscopic examination (Vision-System INV150, Innotech, Anyang, Korea) and tympanometry (Impedance Audiometer AT235, Interacoustics, Middelfart, Denmark), no subjects reported positive history of head injury, ear surgery, audiological and neurological disorders. Hearing thresholds for all subjects were within normal range (less than $20 \mathrm{~dB} \mathrm{HL}$ ) at frequencies of 250 to $8,000 \mathrm{~Hz}$ with a two-channel diagnostic audiometer (Acoustic Analyzer 1200, Starkey, Eden Prairie, MN, USA).

\section{Stimuli and auditory evoked response recording}

AERs were recorded using a GSI Audera system (GrasonStadler, Eden Prairie, MN, USA). For AERs, all subjects were comfortably seated and relaxed in an armchair located in a sound booth. Before electrical placement, the skin was cleaned with alcohol pads and a conducting gel was applied for the connection between the electrode and skin. For the AER recording, electrical responses from both ears were obtained with the active electrode (+) indicating the non-inverting electrode placed on the middle of the forehead $(\mathrm{Fz})$ or vertex $(\mathrm{Cz})$, the reference electrode (-) meaning the inverting electrode placed on the ipsilateral earlobe (A1 or A2), and the ground electrode placed on the low forehead (Fpz). Impedances among three electrodes were less than 5 $\mathrm{k} \Omega$. Electrical responses were elicited by alternating clicks of 0.1 ms durations. The stimuli were presented at $80 \mathrm{~dB}$ normal hearing level (nHL) for ABR and $70 \mathrm{~dB}$ nHL for AMLR through an electromagnetically-shielded insert earphone (ER-3A, Etymotic Research Inc., Elk Grove Village, IL, USA) because these stimulus levels provide strong electrical responses (Choi et al., 2015; Jang et al., 2017). The stimulus rate was changed in 33/s for ABR and 8.0/s for AMLR. The electrical responses were amplified (100,000 times), band-pass filtered from 100 to $3,000 \mathrm{~Hz}$ for ABR and from 10 to $250 \mathrm{~Hz}$ for AMLR, digitalized through an analog to digital converter, and averaged at a sample rate of 2,000 sweeps for each test condition. Analysis time was $15 \mathrm{~ms}$ for ABR and 100 ms for AMLR, and the test duration was 200 seconds.

\section{Data analysis}

Peak and interpeak latencies and peak amplitudes of waves I, 
III, and $\mathrm{V}$ in ABRs and the latencies and amplitudes of $\mathrm{Na}, \mathrm{Pa}$, $\mathrm{Nb}$, and $\mathrm{Pb}$ for AMLR were visually identified and obtained from each subject at different stimulus rates for ABR and AMLR. The latency and amplitude data were compared and analyzed among different conditions. Three independent observers for high reliability confirmed the AER data. All data in the study was reported as a mean \pm standard error. All graphic presentations were made by SigmaPlot (version 9, Systat Software Inc., San Jose, CA, USA).

\section{Statistical analysis}

Statistical differences in the latencies and amplitudes of ABR and AMLR between musicians and non-musicians were also compared using an independent $t$-test (SPSS 19.0, IBM Corp., Armonk, NY, USA). A statistical significance was determined by $p<0.001$, respectively.

\section{RESULTS}

\section{Auditory brainstem responses}

Statistical differences in the absolute latencies of wave I, III, $\mathrm{V}$ in ABR were obtained between students with music training (musicians) and students without music training (non-musicians). The absolute latency of wave I in ABR was 1.44 ( \pm 0.14 ) $\mathrm{ms}$ for musicians and $1.52( \pm 0.14) \mathrm{ms}$ for non-musicians. The absolute latency of wave I was shorter for musician than that for non-musicians. The absolute latency of wave III in ABR was 3.61 $( \pm 0.27) \mathrm{ms}$ for musicians and $3.67( \pm 0.16) \mathrm{ms}$ for non-musicians. The absolute latency of wave III was shorter for musician than that for non-musicians. The absolute latency of wave $\mathrm{V}$ in ABR

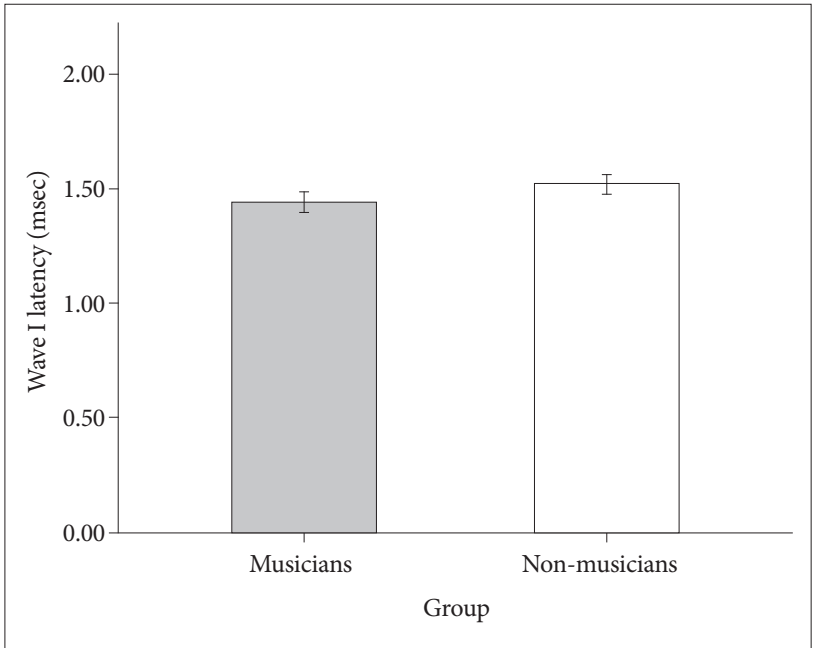

Figure 1. Difference of the absolute latency of wave I between musicians and non-musicians. was $5.11( \pm 0.18) \mathrm{ms}$ for musicians and $5.37( \pm 0.17) \mathrm{ms}$ for nonmusicians. The absolute latency of wave $\mathrm{V}$ was shorter for musician than that for non-musicians. The absolute latencies of wave I, III, and V were shorter for musicians than those for non-musicians.

Statistical differences in the absolute latencies of wave I, III, and $\mathrm{V}$ in $\mathrm{ABR}$ between musicians and non-musicians were performed by an independent $t$-test. Figure 1 shows the absolute latency of wave I in ABR between musicians and non-musicians. For the absolute latency of wave I, there was a significant difference between musicians and non-musicians $[\mathrm{F}(1,78), t=-2.671$, $p<0.01]$. The latency of wave I was significantly shorter for musicians than that for non-musicians.

However, there was no significant difference in the absolute latency of wave III for musicians than for non-musicians. Figure 2 shows the absolute latency of wave $\mathrm{V}$ in ABR between musicians and non-musicians. For the absolute latency of wave $\mathrm{V}$, there was a significant difference between musicians and nonmusicians $[\mathrm{F}(1,78), t=-6.606, p<0.001]$. The latency of wave $\mathrm{V}$ was significantly shorter for musicians than that for non-musicians.

Statistical differences in the interpeak latencies of wave I-III, III-V, and I-V in ABR were obtained between musicians and non-musicians. The interpeak latency of wave I-III in ABR was $2.16( \pm 0.16) \mathrm{ms}$ for musicians and $2.15( \pm 0.29) \mathrm{ms}$ for non-musicians. The interpeak latency of wave I-III was a little longer for musician than that for non-musicians. The interpeak latency of wave III-V in ABR was $1.50( \pm 0.24) \mathrm{ms}$ for musicians and 1.71 $( \pm 0.19) \mathrm{ms}$ for non-musicians. The interpeak latency of wave III-V was shorter for musician than that for non-musicians. The

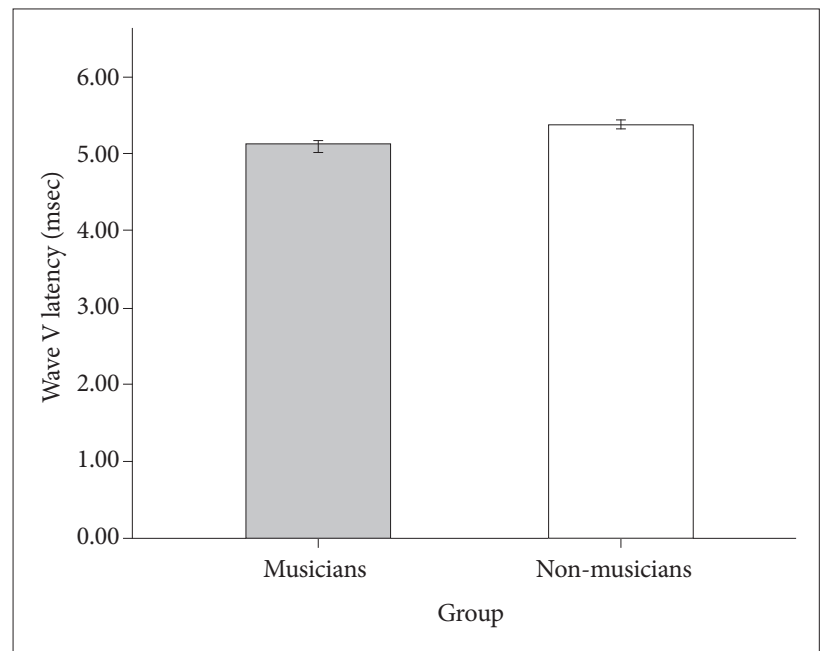

Figure 2. Difference of the absolute latency of wave $V$ between musicians and non-musicians. 


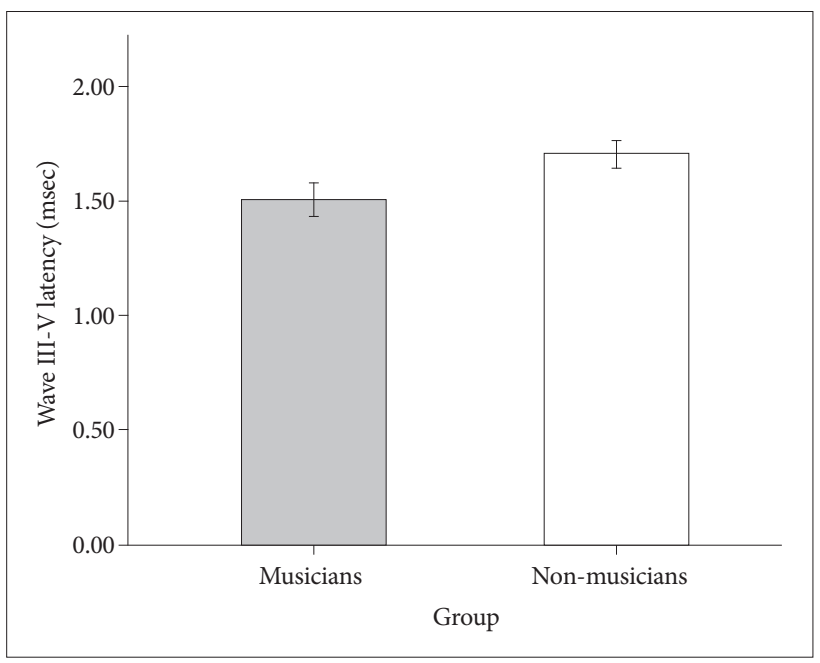

Figure 3. Difference of the interpeak latency of wave III-V between musicians and non-musicians.

interpeak latency of wave I-V in ABR was $3.67( \pm 0.25) \mathrm{ms}$ for musicians and $3.86( \pm 0.20) \mathrm{ms}$ for non-musicians. The interpeak latency of wave I-V was shorter for musician than that for non-musicians. The interpeak latencies of wave III-V and I-V were shorter for musicians than those for non-musicians except those of wave I-III.

Statistical differences in the interpeak latencies of wave I-III, III-V, and I-V in ABR between musicians and non-musicians were performed by an independent $t$-test. There was no significant difference in the interpeak latency of wave I-III for musicians than for non-musicians. Figure 3 shows the interpeak latency of wave III-V in ABR between musicians and non-musicians. For the interpeak latency of wave III-V, there was a significant difference between musicians and non-musicians $[\mathrm{F}(1,78), t=$ $-4.126, p<0.001]$. The interpeak latency of wave III-V was significantly shorter for musicians than that for non-musicians.

Figure 4 shows the interpeak latency of wave I-V in ABR between musicians and non-musicians. For the interpeak latency of wave I-V, there was a significant difference between musicians and non-musicians $[\mathrm{F}(1,78), t=-3.705, p<0.001]$. The interpeak latency of wave I-V was significantly shorter for musicians than that for non-musicians.

Statistical differences in the amplitudes of wave I, III, V in ABR were obtained between musicians and non-musicians. The amplitude of wave I in ABR was $0.180( \pm 0.074) \mu \mathrm{V}$ for musicians and $0.153( \pm 0.090) \mu \mathrm{V}$ for non-musicians. The amplitude of wave I was larger for musician than that for non-musicians. The amplitude of wave III in ABR was $0.250( \pm 0.091) \mu \mathrm{V}$ for musicians and $0.290( \pm 0.307) \mu \mathrm{V}$ for non-musicians. The amplitude of wave III was smaller for musician than that for non-musicians.

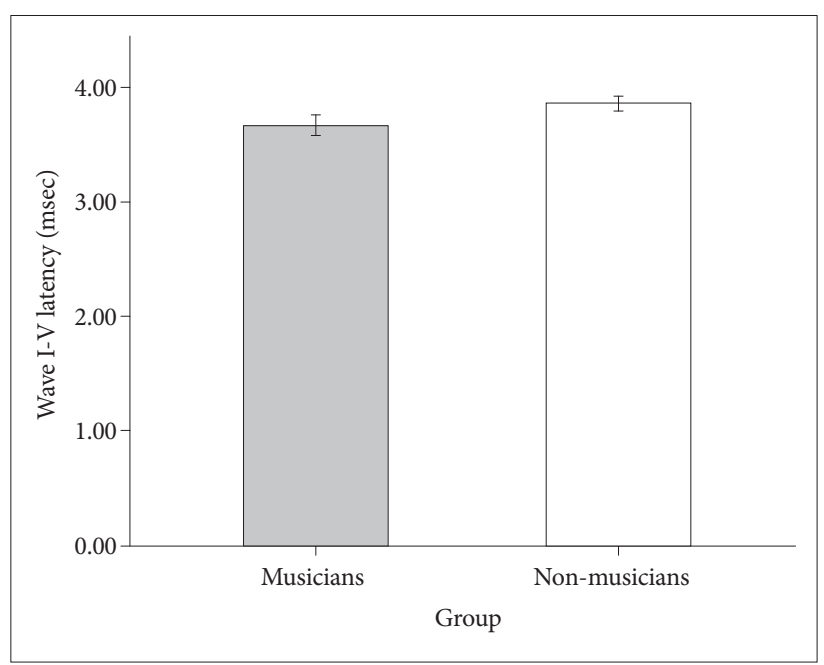

Figure 4. Difference of the interpeak latency of wave I-V between musicians and non-musicians.

The amplitude of wave $\mathrm{V}$ in $\mathrm{ABR}$ was $0.249( \pm 0.117) \mu \mathrm{V}$ for musicians and $0.241( \pm 0.227) \mu \mathrm{V}$ for non-musicians. The amplitude of wave $\mathrm{V}$ was larger for musician than that for non-musicians.

Statistical differences in the amplitudes of wave I, III, and V in $\mathrm{ABR}$ between musicians and non-musicians were performed by an independent $t$-test. There was no significant difference in the amplitude of wave I, III, and V for musicians than for nonmusicians.

\section{Auditory middle latency response}

Statistical differences in the latencies and amplitudes of $\mathrm{Na}$, $\mathrm{Pa}, \mathrm{Nb}$, and $\mathrm{Pb}$ of AMLR were obtained between musicians and non-musicians. The absolute latency of $\mathrm{Na}$ was $17.45( \pm 2.18)$ $\mathrm{ms}$ for musicians and $18.72( \pm 2.17) \mathrm{ms}$ for non-musicians. The absolute latency of $\mathrm{Na}$ was shorter for musician than that for nonmusicians. The absolute latency of Pa was $29.90( \pm 2.00) \mathrm{ms}$ for musicians and $29.62( \pm 2.47) \mathrm{ms}$ for non-musicians. The absolute latency of $\mathrm{Pa}$ was a little longer for musician than that for non-musicians. The absolute latency of $\mathrm{Nb}$ was $41.55( \pm 2.65) \mathrm{ms}$ for musicians and $42.50( \pm 3.58) \mathrm{ms}$ for non-musicians. The absolute latency of $\mathrm{Nb}$ was shorter for musician than that for nonmusicians. The absolute latency of Pb was 60.67 ( \pm 5.38) $\mathrm{ms}$ for musicians and $60.63( \pm 4.55)$ ms for non-musicians. The absolute latencies of $\mathrm{Pb}$ were longer for musicians than those for nonmusicians.

Statistical differences in the absolute latencies of $\mathrm{Na}, \mathrm{Pa}, \mathrm{Nb}$, and $\mathrm{Pb}$ in AMLR between musicians and non-musicians were performed by an independent $t$-test. Figure 5 shows the absolute latency of $\mathrm{Na}$ between musicians and non-musicians. For the absolute latency of $\mathrm{Na}$, there was a significant difference between 


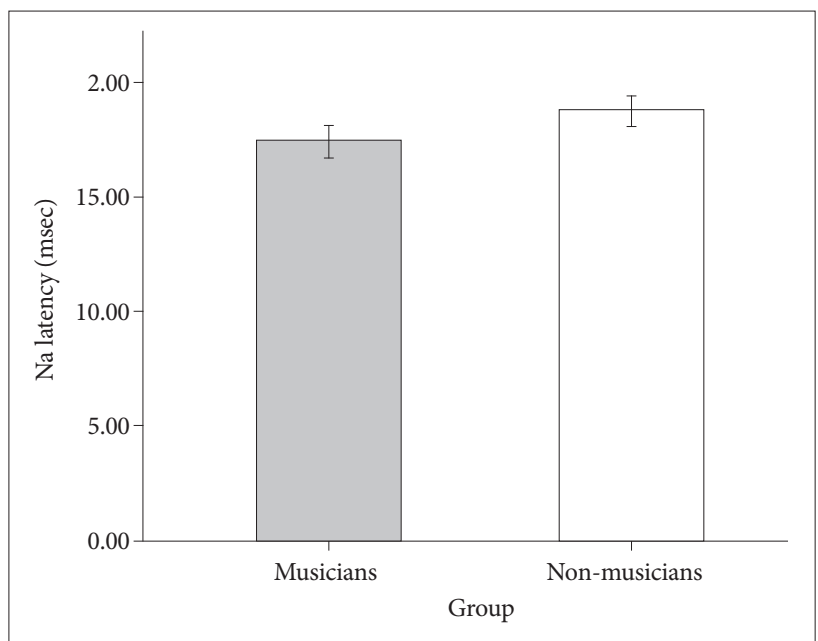

Figure 5. Difference of the latency of $\mathrm{Na}$ between musicians and non-musicians.

musicians and non-musicians $[\mathrm{F}(1,78), t=-2.607, p<0.05]$. The latency of $\mathrm{Na}$ was significantly shorter for musicians than that for non-musicians. However, there were no significant differences in the absolute latency of $\mathrm{Pa}, \mathrm{Nb}$, and $\mathrm{Pb}$ in AMLR for musicians than for non-musicians.

Statistical differences in the amplitudes of $\mathrm{Na}, \mathrm{Pa}, \mathrm{Nb}$, and $\mathrm{Pb}$ of AMLR were obtained between musicians and non-musicians. The amplitude of $\mathrm{Na}$ was $-1.20( \pm 1.55) \mu \mathrm{V}$ for musicians and $-1.60( \pm 1.68) \mu \mathrm{V}$ for non-musicians. The amplitude of $\mathrm{Na}$ was smaller for musician than that for non-musicians. The amplitude of $\mathrm{Pa}$ was $0.56( \pm 0.45) \mu \mathrm{V}$ for musicians and $0.50( \pm 0.36)$ $\mu \mathrm{V}$ for non-musicians. The amplitude of $\mathrm{Pa}$ was a little larger for musician than that for non-musicians. The amplitude of $\mathrm{Nb}$ was $-0.52( \pm 0.35) \mu \mathrm{V}$ s for musicians and $-0.50( \pm 0.32) \mu \mathrm{V}$ for nonmusicians. The amplitude of $\mathrm{Nb}$ was a little larger for musician than that for non-musicians. The amplitude of $\mathrm{Pb}$ was $0.43( \pm$ 0.36) $\mu \mathrm{V}$ for musicians and $0.38( \pm 0.33) \mu \mathrm{V}$ for non-musicians. The amplitude of $\mathrm{Pb}$ was larger for musicians than those for non-musicians. There were no significant differences in the amplitudes of $\mathrm{Na}, \mathrm{Pa}, \mathrm{Nb}$, and $\mathrm{Pb}$ in AMLR for musicians than for non-musicians.

\section{DISCUSSIONS}

This study investigated the effect of music training on neuroplasticity by comparing the absolute latencies and amplitude of wave I, III, and V in ABR between college students with music training (musicians) and college students without music training (non-musicians). There were significant differences in the absolute latencies of wave I and V in ABR and in the interpeak latencies of wave III-V and I-V between musicians and nonmusicians. In musician showed shorter latency of waves I and $\mathrm{V}$ and shorter interpeak latencies of wave III-V and I-V in ABR than that for non-musicians. Although there was no significant difference in the absolute latency of wave III, musician showed shorter latency than non-musicians. In spite of no significant differences, the amplitudes of wave I and V were greater in musicians than those in non-musicians whereas the amplitude of wave III was smaller in musicians than that in non-musicians. The wave I in ABR comes from the dorsal portion of the auditory nerve whereas the wave III and V originates in or near the cochlear nucleus and at the termination of the lateral lemniscus fiber which is contralateral to the stimulated ear, respectively (Møller et al., 1995).

On the other hand, comparing the latencies and amplitudes of $\mathrm{Na}, \mathrm{Pa}, \mathrm{Nb}$, and $\mathrm{Pb}$ in AMLR between musicians and nonmusicians, there was a significant difference in the latency of $\mathrm{Na}$ in AMLR. The Na component of AMLR arises mostly from subcortical structures with prominent contributions from the inferior colliculus within the midbrain regions or the thalamus (McGee et al., 1991). The results indicate that music training primarily activates subcortical structures as well as cortical structures.

In summary, our current study showed reduced latencies of wave I and V in ABR, decreased interpeak latencies of wave III-V and $\mathrm{I}-\mathrm{V}$ in $\mathrm{ABR}$, and reduced latency of $\mathrm{Na}$ in AMLR for musicians. These indicate that music training enhances electrophysiological responses in musicians. Although our study showed reduced latencies of ABR wave I and $\mathrm{V}$ and $\mathrm{Na}$ of AMLR in musicians, another study reported that the mean latencies of waves I, III, and V of ABR in pop/rock musicians were shorter than those in non-musicians (Samelli et al., 2012). This difference may result from different types of musicians, training period, and different exposure degree to noise. In addition, the different results in AERs among studies may be affected by a lot of different factors such as period, involvement, intensity, repetition, and frequency of music training (Green \& Bavelier, 2008; Kleim \& Jones, 2008).

Based on the results of our current research, it was observed that the main areas of the brainstem and the primary cortices affected by music training are consistent with other studies (Sanju \& Kumar, 2016; Wong et al., 2007). Unlike other everyday activities, music training involves broad brain areas from the brainstem to the cortical regions and requires simultaneous integration of multimodalities from sensory modality such as audition, vision to motor control as well as high-order cognitive 
processes, memory, and learning. Music training is a highly complex task consisting of physical and mental operations such as the translation of visually presented musical symbols into complex, sequential finger movements, improvisation, memorization of musical phrases, and identification of tones without a reference tone (Gaser \& Schlaug, 2003). Therefore, music demands cognitive and neural challenges requiring cooperation of many modalities and actions. Compared to non-musicians, musicians have enhanced auditory perception, increased hearing abilities, a strong connection between conceptual brain systems and auditory perceptual skills, enhanced temporal resolution, and increased frequency resolution (Bidelman \& Alain, 2015; Kumar et al., 2016; Sanju \& Kumar, 2016). Activation in the brain of musicians was observed in auditory cortex, adjacent areas in the superior temporal sulcus, right posterior superior temporal gyrus, and the upper part of middle temporal gyrus (Hoenig et al., 2011).

Our current study suggests an important implication which can be applied to auditory training or other rehabilitation programs. Musical training can be used an useful tool to enhance brainstem, cortical and subcortical encoding of speech (Sanju \& Kumar, 2016), pre-attentive and attentive auditory discrimination skills in a variety of clinical populations (Putkinen et al., 2014), plasticity of the brain, cognitive skills (Sanju \& Kumar, 2016), linguistic skills such as phonological awareness, dynamic acoustic analysis, reading, pitch and lexical stress processing and speech-language proficiency, working memory, and sequencing process (Kraus \& Chandrasekaran, 2010; Tallal \& Gaab, 2006). This usefulness of musical training may result in improvement of speech perception in various populations and environments.

\section{Ethical Statement}

The experimental procedures and methods were reviewed and approved by the Bioethic Committee of the Catholic University of Daegu (CUIRB-2017-0081).

\section{Acknowledgments N/A}

\section{Declaration of Conflicting Interests}

There are no conflict of interests.

\section{Funding}

N/A

\section{Author Contributions}

All authors contributed equally to this work. C.C. designed and performed experiments, analyzed data, and wrote the paper; H.C. designed and performed experiments in the clinic; C.C. and H.C. provided statistical analysis and critical revision; C.C. and H.C. designed experiments, analyzed data, and wrote the paper. Also, the authors discussed the results together and implications and commented on the manuscript at each stage.

\section{ORCID iD}

Chul-Hee Choi https://orcid.org/0000-0003-1844-3072

\section{REFERENCES}

Anderson, S. \& Jenkins, K. (2015). Electrophysiologic assessment of auditory training benefits in older adults. Seminars in Hearing, 36(4), 250262.

Bidelman, G. M. \& Alain, C. (2015). Musical training orchestrates coordinated neuroplasticity in auditory brainstem and cortex to counteract age-related declines in categorical vowel perception. Journal of Neuroscience, 35(3), 1240-1249.

Bidelman, G. M. \& Krishnan, A. (2010). Effects of reverberation on brainstem representation of speech in musicians and non-musicians. Brain Research, 1355, 112-125.

Choi, C. H., Jang, K. H., \& Choi, S. H. (2015). Effect of stimulus rate and gender on auditory brainstem response in Korean young adults. Audiology, 11(2), 140-155.

Ericsson, K. A., Krampe, R. T., \& Tesch-Romer, C. (1993). The role of deliberate practice in the acquisition of expert performance. Psychological Review, 100(3), 363-406.

Gaser, C. \& Schlaug, G. (2003). Brain structures differ between musicians and non-musicians. Journal of Neuroscience, 23(27), 9240-9245.

Green, C. S. \& Bavelier, D. (2008). Exercising your brain: A review of human brain plasticity and training-induced learning. Psychology and Aging, 23(4), 692-701.

Herholz, S. C. \& Zatorre, R. J. (2012). Musical training as a framework for brain plasticity: Behavior, function, and structure. Neuron, 76(3), 486502.

Hoenig, K., Müller, C., Herrnberger, B., Sim, E. J., Spitzer, M., Ehret, G., et al. (2011). Neuroplasticity of semantic representations for musical instruments in professional musicians. NeuroImage, 56(3), 1714-1725.

Jang, K. H., Choi, S. H., \& Choi, C. H. (2017). Effect of stimulus rate and gender on auditory middle latency response in young adults. Audiology and Speech Research, 13(2), 108-114.

Kleim, J. A. \& Jones, T. A. (2008). Principles of experience-dependent neural plasticity: Implications for rehabilitation after brain damage. Journal of Speech, Language, and Hearing Research, 51(1), S225-S239.

Kraus, N. \& Chandrasekaran, B. (2010). Music training for the development of auditory skills. Nature Reviews Neuroscience, 11(8), 599-605.

Kumar, P., Sanju, H. K., \& Nikhil, J. (2016). Temporal resolution and active auditory discrimination skill in vocal musicians. International Archives of Otorhinolaryngology, 20(4), 310-314.

McGee, T., Kraus, N., Comperatore, C., \& Nicol, T. (1991). Subcortical and cortical components of the MLR generating system. Brain Research, 544(2), 211-220.

Miyamae, T. (2018). Musical experience and brain plasticity: Does musical training change brain structure and function? Brain Nerve, 70(6), 617-631.

Møller, A. R., Jho, H. D., Yokota, M., \& Jannetta, P. J. (1995). Contribution from crossed and uncrossed brainstem structures to the brainstem auditory evoked potentials: A study in humans. Laryngoscope, 105(6), 596-605.

Pantev, C. \& Herholz, S. C. (2011). Plasticity of the human auditory cortex related to musical training. Neuroscience and Biobehavioral Reviews, 35(10), 2140-2154.

Pantev, C., Lappe, C., Herholz, S. C., \& Trainor, L. (2009). Auditory-somatosensory integration and cortical plasticity in musical training. Annals of the New York Academy of Sciences, 1169, 143-150.

Parbery-Clark, A., Skoe, E., \& Kraus, N. (2009). Musical experience limits the degradative effects of background noise on the neural processing of sound. Journal of Neuroscience, 29(45), 14100-14107.

Parbery-Clark, A., Strait, D. L., \& Kraus, N. (2011). Context-dependent encoding in the auditory brainstem subserves enhanced speech-in-noise 
perception in musicians. Neuropsychologia, 49(12), 3338-3345.

Putkinen, V., Tervaniemi, M., Saarikivi, K., de Vent, N., \& Huotilainen, M. (2014). Investigating the effects of musical training on functional brain development with a novel melodic MMN paradigm. Neurobiology of Learning and Memory, 110, 8-15.

Samelli, A. G., Matas, C. G., Carvallo, R. M., Gomes, R. F., de Beija, C. S., Magliaro, F. C., et al. (2012). Audiological and electrophysiological assessment of professional pop/rock musicians. Noise and Health, 14(56), 6-12.

Sanju, H. K. \& Kumar, P. (2016). Enhanced auditory evoked potentials in musicians: A review of recent findings. Journal of Otology, 11(2), 63-72. Strait, D. L., O'Connell, S., Parbery-Clark, A., \& Kraus, N. (2014). Musicians' enhanced neural differentiation of speech sounds arises early in life: Developmental evidence from ages 3 to 30. Cerebral Cortex, 24(9), 2512-2521.

Tallal, P. \& Gaab, N. (2006). Dynamic auditory processing, musical experience and language development. Trends in Neurosciences, 29(7), 382-390.

Wong, P. C., Skoe, E., Russo, N. M., Dees, T., \& Kraus, N. (2007). Musical experience shapes human brainstem encoding of linguistic pitch patterns. Nature Neuroscience, 10(4), 420-422. 